\title{
Multiple mechanisms of germ cell loss in the perinatal mouse ovary
}

\author{
Patricia Rodrigues ${ }^{1,2}$, Darlene Limback ${ }^{1}$, Lynda K McGinnis ${ }^{1}$, Carlos E Plancha ${ }^{2}$ \\ and David F Albertini ${ }^{1,3}$ \\ ${ }^{1}$ Department of Molecular and Integrative Physiology, Center for Reproductive Sciences, 3088 Kansas Life Science \\ Innovations Center, University of Kansas Medical Center, KLSIC Room 3016, 3901 Rainbow Boulevard, Kansas City, \\ Kansas 66160, USA, ${ }^{2}$ Unidade de Biologia da Reprodução, Instituto de Medicina Molecular and Instituto de \\ Histologia e Biologia do Desenvolvimento, Faculdade de Medicina da Universidade de Lisboa, Avenue Professor Egas \\ Moniz, 1649-028 Lisbon, Portugal and ${ }^{3}$ Marine Biological Laboratories, Woods Hole, Massachusetts 02543, USA \\ Correspondence should be addressed to D F Albertini; Email: dalbertini@kumc.edu
}

\begin{abstract}
In the perinatal ovary of most mammals, external and internal factors establish a primordial follicle reserve that specifies the duration of the reproductive lifespan of a given species. We analyzed the mechanism of follicle loss and survival in C57BI/6 mice using static and dynamic assays of apoptosis, autophagy, and ovarian morphogenesis. We confirm an initial loss soon after birth, when about $44 \%$ of the germ cells detectable at the end of the fetal period abruptly disappear. The observations that (1) few germ or somatic cells were apoptotic in newborn ovaries, (2) vitally stained organ cultures exhibit active extrusion of non-apoptotic germ cells and (3) germ-cell lysosome amplification occurs at birth suggested that additional mechanisms are involved in perinatal germ cell loss. Newborn mouse ovaries cultured in the pH sensitive dye lysotracker red exhibit an increased incidence of acidified non-apoptotic germ cells when maintained in the absence but not in the presence of serum, implying a role for autophagy in germ cell attrition. Inhibitors of autophagy, but not apoptosis, reduce germ cell acidification induced by serum starvation in ovary organ cultures and protein mediators of both autophagy and apoptosis are expressed at birth. From these findings we suggest that multiple perinatal mechanisms establish the primordial follicle reserve in mice.
\end{abstract}

Reproduction (2009) 137 709-720

\section{Introduction}

The transition from fetal to neonatal life is a critical phase of normal development in eutherian mammals during which organ systems adapt to post-parturition starvation. Many organ systems in newborns adjust progenitor cell density and function by engaging apoptotic and non-apoptotic mechanisms of programmed cell death (PCD; Edinger \& Thompson 2004). There are three types of PCD. Type I, or apoptosis, is characterized morphologically by nuclear condensation and fragmentation, cell shrinkage and membrane blebbing (Tilly 2001, Edinger \& Thompson 2004). Type II, or autophagy, is a lysosomal degradation pathway that involves formation of autophagic vacuoles during periods of massive cell elimination (Edinger \& Thompson 2004, Qu et al. 2007). Type III, or necrosis, is characterized by plasma membrane breakdown causing an inflammatory reaction (Edinger \& Thompson 2004). Apoptosis is coupled to autophagy as a means to maintain tissue viability and energy homeostasis in developing tissues during the demanding neonatal phase in mammals (Kuma et al. 2004, Yu et al. 2004, Lum et al. 2005). This coupling occurs either via constitutive pathways, where lysosomes degrade the apoptotic bodies, or through an alternative pathway in which lysosomal proteases such as cathepsins, trigger apoptosis (Bursch 2001, Guicciardi et al. 2004). Amongst the organ systems that exhibit profound cell loss at birth is the ovary, where it has long been recognized that massive female germ cell attrition post partum precedes the establishment of a fixed follicle reserve that is progressively depleted during the reproductive lifespan (Reynaud \& Driancourt 2000, Tilly 2001, Qu et al. 2007). Studies favoring a major role for germ cell apoptosis include those using ubiquitously targeted deletion of genes involved in Type I PCD. Accordingly, an increase or decrease in follicle numbers has been reported in postnatal mice bearing systemically targeted gene deletions for antiapoptotic, or pro- apoptotic genes (Perez et al. 1999). Although apoptosis is believed to account for the bulk of germ cell loss in fetal mouse ovaries (Coucouvanis et al. 
1993, De Pol et al. 1997, Pepling \& Spradling 2001), other processes such as germ cell extrusion (Wordinger et al. 1990) and autophagy also participate in preand postpartum adjustments of germ cell numbers (Wordinger et al. 1990, Lobascio et al. 2007). Both apoptosis (Edinger \& Thompson 2004, Maiuri et al. 2007) and autophagy (Maiuri et al. 2007), lysosomemediated PCD processes (Guicciardi et al. 2004), have been proposed to serve cooperatively during developmental transitions in other organs, but this prospect has yet to be studied during perinatal ovarian germ cell loss.

The present study addresses several questions related to morphogenesis of the mouse ovary revealing a previously unappreciated level of complexity in PCD that sets and maintains adequate numbers of follicles for reproductive function in mice without invoking replacement strategies. Our findings suggest that multiple mechanisms including autophagy mediate germ cell loss and are used in a coordinated and developmentally regulated fashion.

\section{Results}

\section{Characterization of germ cell number during ovarian development}

Given the wide range of variability reported for germ cells during ovarian development in mice, we adopted a technical modification for our morphometric analyses. Here, histological sections processed for detection of mouse vasa homologue (MVH), a specific germ cell marker (Fujiwara et al. 1994) combined with periodic acid Schiff's (PAS) staining so as to render extracellular matrix, including the zona pellucida, clearly discernible (Fig. 1). Sections of ovaries from C57Bl/6 females ranging in age from embryonic day (E) 15.5 through to postnatal day $(\mathrm{P}) 150$ were morphometrically analyzed for total oocyte, follicle, and/or zona pellucida profiles
(Fig. 2 and Supplementary Table 1, which can be viewed online at www.reproduction-online.org/supplemental/). Examples of embryonic, postnatal, and prepubertal ovaries are shown in Fig. 1 to emphasize the striking histological transformation that takes place over these key developmental stages that include resolution of ovigerous cords into solitary follicles (Fig. 1A and B) and expansion of somatic cells including development of the vasculature (Fig. 1C).

In this study, we confirm the decrease in mean germ cell numbers per ovary at birth, with $5783 \pm 475$ (confidence intervals (Cl): 4463-7103) total follicles observed prior to birth (E19.5) and 3252 \pm 820 (Cl: 9765529) total follicles after birth (P2; Fig. 2 and Supplementary Table 1). Variations in mean oocyte number were also evident from immediately after birth through to the onset of reproductive cyclicity (data not shown). It should be emphasized that although germ cell number between P2 and P20 remains unchanged, considerable variability is observed between animals as expressed by the wide $\mathrm{Cl}$ found for the mean numbers of total follicles at ages P2 (Cl: 976-5529), 12 (Cl: 264-4996) and 20 (Cl: 947-5399; Fig. 2). In sharp contrast to the lack of stability in germ cell number observed pre-pubertally, the rate of decline in follicle number from the onset of cyclicity (P42, Cl: 1830-3713 total follicles) through adulthood is consistent between time points and animals. This was further confirmed by performing similar analysis on animals in which stages of the estrous cycle were monitored (data not shown). Again, germ cell counts, corpora lutea and atretic follicles were similar at the age evaluated (P100, Cl: 1419-2579 total follicles). Furthermore, estimates of the total number of zona pellucida remnants as a function of animal age also showed that their appearance coincided with the onset of reproductive cyclicity, from undetectable at P20 to $20.8 \pm 3.2$ at P42 and $34.9 \pm 1.7$ at P100 (mean number per ovary for $n=5$ animals for each time point). These
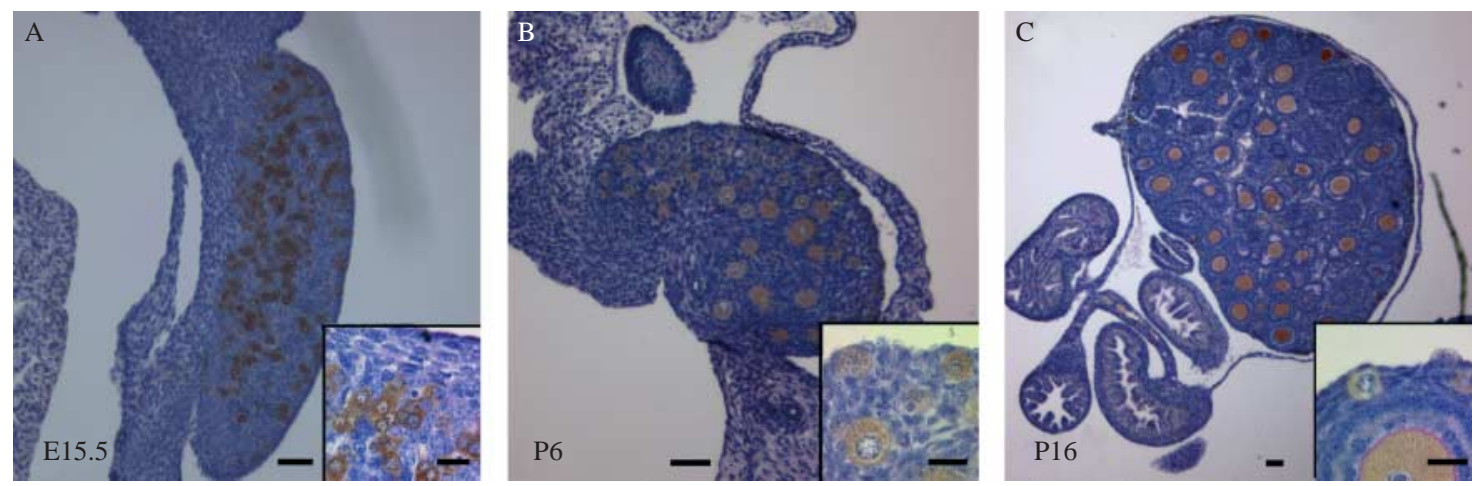

Figure 1 Germ cell disposition during ovarian histogenesis. MVH immunocytochemistry (brown reaction product) defines developmental variance in germ cell density and location. (A) Embryonic day 15.5 ovary, showing germ cell clusters and relative paucity of somatic tissue (inset). (B) Postnatal day 6 ovary, with cortical primordial follicles and medullary primary follicles; note apposition of peritoneal mesothelium to ovarian surface defining bursal cavity. (C) Postnatal day 16 ovary illustrating onset of antrum formation in centrally located follicles and cortically disposed preantral follicles with growing oocytes; inset shows 2 primordial follicles near surface and subtended by a primary follicle. Scale bars $=40 \mu \mathrm{m}$ for $\mathrm{A}$, B and C, whereas for insets scale bars $=20 \mu \mathrm{m}$. 


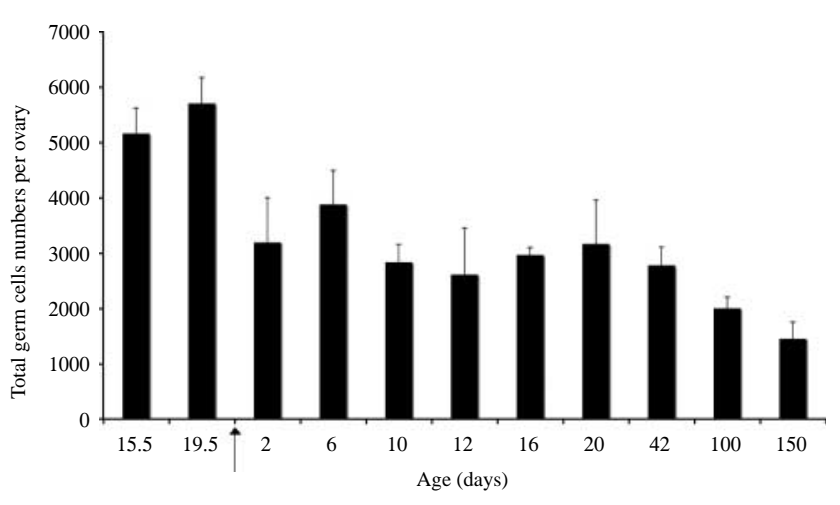

Figure 2 Mean number of total germ cell number per ovary from embryonic day 15 (E15.5) through to adult day 150 (P150). Note marked decrease in mean germ cell number following birth $(P<0.05)$, and animal-to-animal variance in oocyte density between days P6 and P20 of age. Onset of cyclicity (P42) coincides with decrease in mean germ cell numbers that steadily declines up to P150. Data are presented as mean \pm S.E.M. ( $n=5$ animals per age group). Arrow indicates day of birth (day 20 post coitum).

results are consistent with little if any net change in follicle number during prepubertal life and therefore prompted further enquiry into the nature of germ cell loss at perinatal developmental transitions.

\section{Germ cell apoptosis during pre- and postnatal ovary remodeling}

We first investigated the expression of various apoptosis markers prior to and following birth. Two common markers for early apoptosis, active caspase 3 and cleaved poly (ADP ribose) polymerase (PARP), were evaluated and the more traditional TUNEL assay for terminal stages of apoptosis. E15.5 and E19.5 samples exhibited limited staining for all markers in germ and somatic cells (Fig. 3A-C). This relative paucity of apoptotic germ cells was also evident in postnatal samples (Fig. 3D-F). As shown below, large clusters of germ cells were often seen at the surface of P2 ovaries, but even these clearly discarded oocytes were only rarely found to be positive for apoptotic markers. In contrast, ovaries from adult cycling females exhibited the apoptosis patterns well established for mammalian ovary (Fig. 3G-I), with granulosa cells (GCs), but not oocytes in atretic follicles positive for apoptosis markers. The rarity of apoptotic germ cells prior to and following birth is difficult to reconcile with the $40 \%$ reduction seen by us and others and prompted investigation of alternative cell death mechanisms.

\section{Postnatal ovarian histogenesis and germ cell extrusion}

We first evaluated germ cell extrusion or shedding, as a mechanism of germ cell loss. Oogonia and primordial follicles were scored based on their position relative to the ovarian epithelium; these were classified as extraovarian if in the bursa cavity or intra-epithelial if positioned at the ovarian surface (Fig. 4A-D). As summarized in Fig. 4E, the mean number of extraovarian oogonia/oocytes observed in the bursa account for a minor fraction $(\sim 2 \%)$ of the total germ cells recorded at these time points. Intra-epithelial oogonia/ oocytes represent a transient population of about 300 follicles at each stage examined up to P42, after which time they are not detected (Fig. 4E). Because of the difficulty in determining the flux rate of oocyte extrusion in fixed samples, we conducted live imaging studies in ovarian organ cultures. Intact E19.5, P0 (day of birth), and P1 ovaries were cultured for 4 or $18 \mathrm{~h}$. Germ cells associated with the ovarian surface were large and spherical and rarely contained associated somatic cells (Supplementary Figure 1, which can be viewed online at www.reproduction-online.org/supplemental/) whereas those that accumulated on the bottom of the culture dish after $4 \mathrm{~h}$ in culture were found in clusters of 4-8 cells (Supplementary Figure 1). While active extrusion was observed at ages examined, attempts to calculate a rate of loss were unsuccessful. An estimated extrusion rate of 150 germ cells per ovary over an $18 \mathrm{~h}$ culture interval for P0 ovaries was obtained (Supplementary Figure 1) but in the absence of a way to follow single germ cells, we conclude that this mechanism of loss accounts for only a minor fraction of the loss observed until a more systematic analysis can be performed.

\section{Lysosome compartment amplification and autophagy}

Since lysosomes are involved in both autophagy and apoptosis (Guicciardi et al. 2004), we first analyzed their expression in somatic and germ cells of perinatal ovaries. Using LAMP1 antibody, which detects a membrane constituent of functional lysosomes, a striking increase in lysosome density was observed from E19.5 to post-natal ovaries (Fig. 5A and B). Figure 5C and D show further that the increase in LAMP1 staining in germ cells coincides with the onset of oocyte growth and follicle assembly. Quantification of LAMP1 by image analysis of stained ovarian sections further confirmed the lysosome amplification post-birth (Fig. 5E). Significant changes in the amplification of lysosomes were observed from E19.5 2.02 \pm 0.21 (Cl: 1.60-2.43) to P2 15.08 \pm 0.95 (Cl: 13.19-16.19) by measuring the mean LAMP1 staining per germ cell. The general pattern of increased lysosome expression after birth was also seen with the vital lysosome marker acridine orange (data not shown). Thus, lysosome amplification in all oocytes occurs upon birth and as in other postnatal tissues may represent an adaptive response to postnatal starvation. To directly address this question, an additional experimental strategy was used to ascertain the relative contributions of apoptosis and autophagy in the perinatal mouse ovary. 

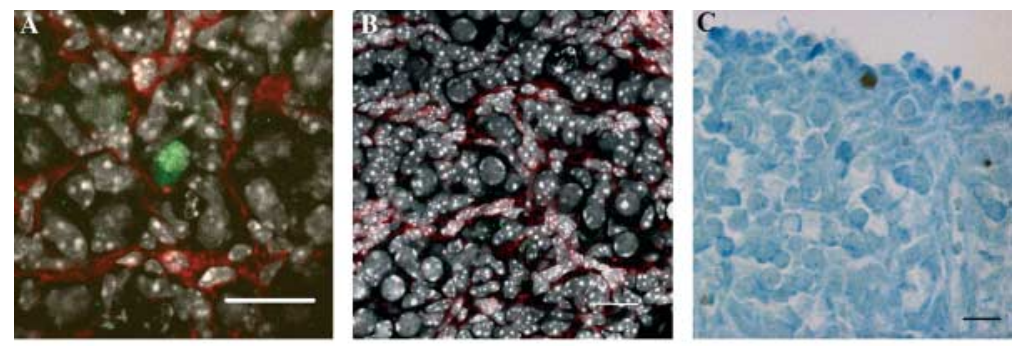

E19.5
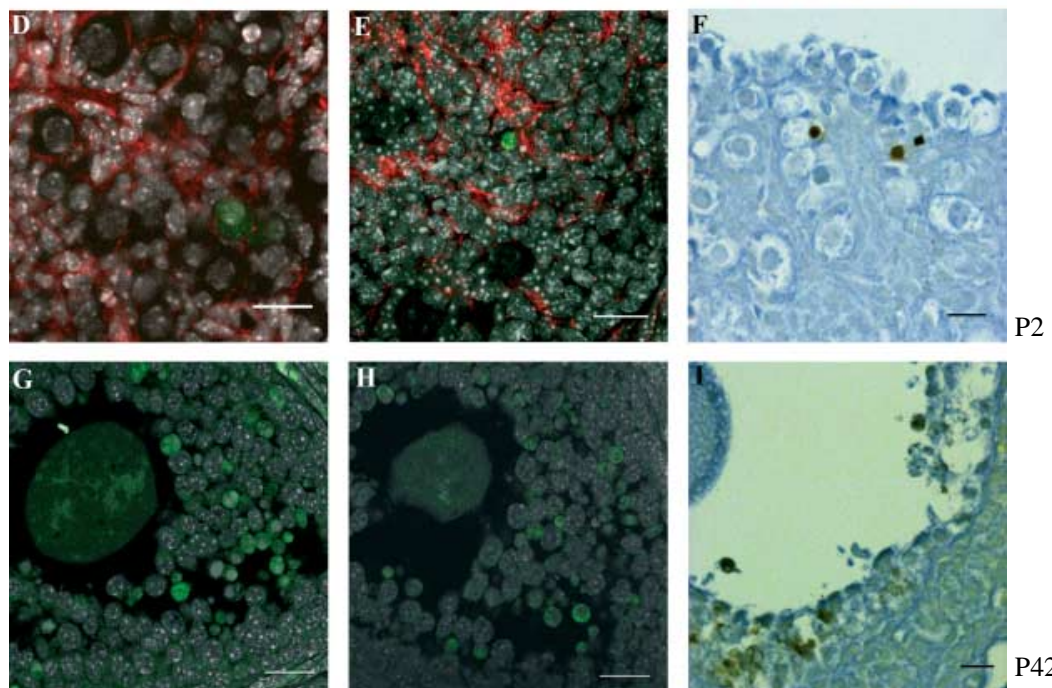

PARP

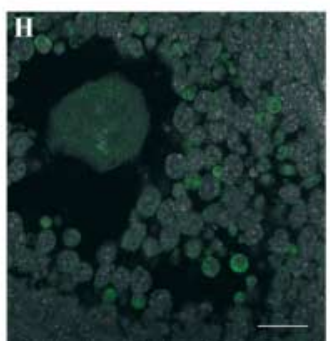

Caspase 3

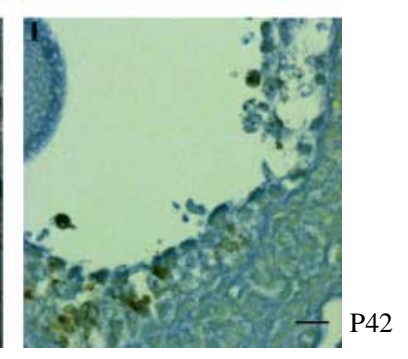

TUNEL
Figure 3 Pre- and postnatal apoptosis patterns. Comparison of apoptosis detection by cleaved$\operatorname{PARP}(A, D$, and $G)$, active caspase $3(B, E$, and $H$ ) or TUNEL (C, F, and I) assays in E19.5 (A, B, and C), $\mathrm{P} 2$ post partum $(\mathrm{D}, \mathrm{E}$, and $\mathrm{F})$, and $\mathrm{P} 42(\mathrm{G}, \mathrm{H}$, and $\mathrm{I})$ C57Bl/6 ovaries. (A) Confocal optical section of intact E19.5 ovary showing a solitary cleavedPARP positive (green) germ cell amongst many negative germ cells ( $f$-actin red, nuclei white). (D) Confocal optical section of intact ovary (P2) showing absence of staining in germ (one exception) or somatic cells for cleaved-PARP. Also note rare TUNEL positive cells in comparable ovaries (B, E). (G) (PARP red, GDF9 green), (H) (caspase red) and (I) (TUNEL brown) show that under identical processing conditions, apoptosis is readily demonstrable in granulosa cells of antral follicles in day P42 ovary. Scale bars $=20 \mu \mathrm{m}$.
We adapted the methods of Zucker et al. (1998) to image intact ovaries that had been labeled with the probe LysoTracker Red (LTR) using high-resolution confocal microscopy to monitor cytoplasmic and organellar acidification in tissues in an unperturbed state. In E19.5 ovaries, LTR reveals smaller apoptotic cells, macrophages identified by multiple lysosomes, and larger germ cells evidenced by acidified cytoplasm (Fig. 6A and B). Acidified LTR labeled germ cells were common at P2, but rare at later stages of ovarian
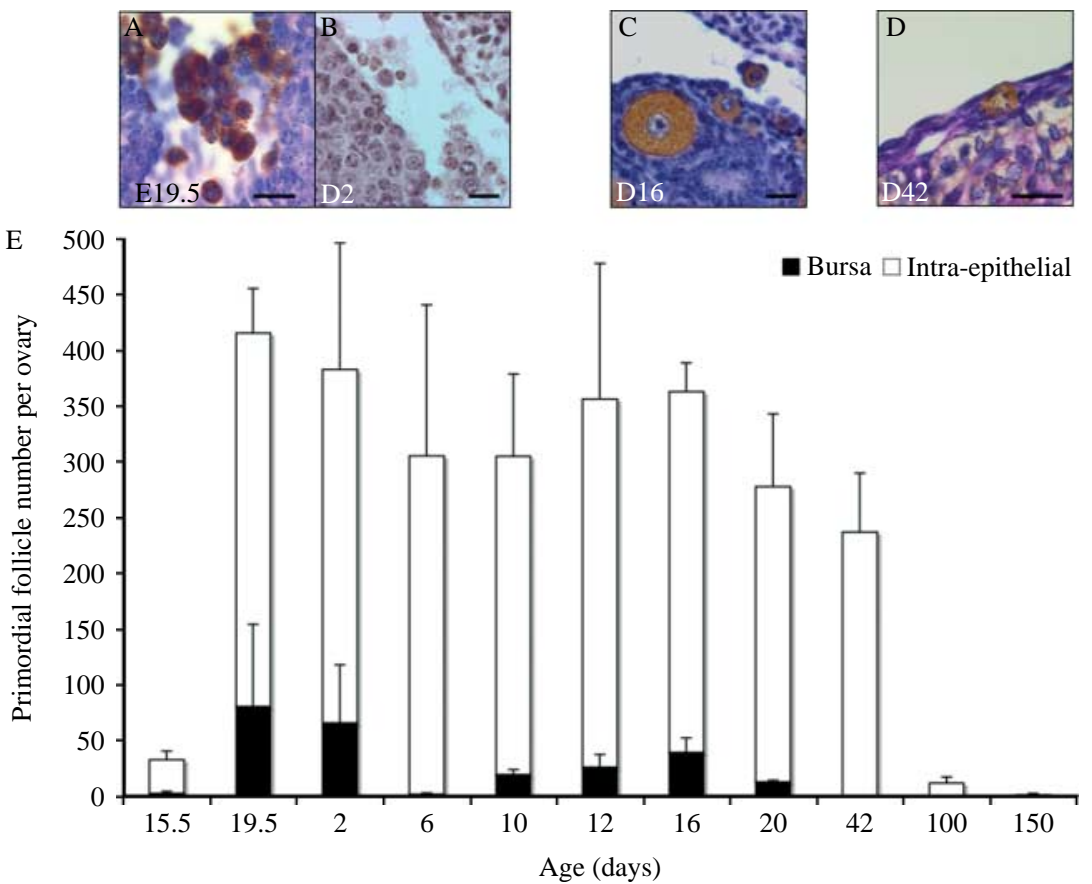

Figure 4 Developmental pattern of germ cell extrusion in mouse ovary. Germ cells were classified as extra-ovarian when within the bursal cavity (A-C) or intraepithelial when associated with or just beneath ovarian surface (D). (A-D) Bright field images of sections stained with MVH (brown), hematoxylin (blue) and PAS (pink). Bars $=20 \mu \mathrm{m}$. (E) Comparison of extra-ovarian (filled bars) or intraepithelial (open bars) germ cells over developmental age range studied (E15.5P150). Mean number primordial follicles \pm S.E.M. per time point are plotted ( $n=5$ ovaries/age). 

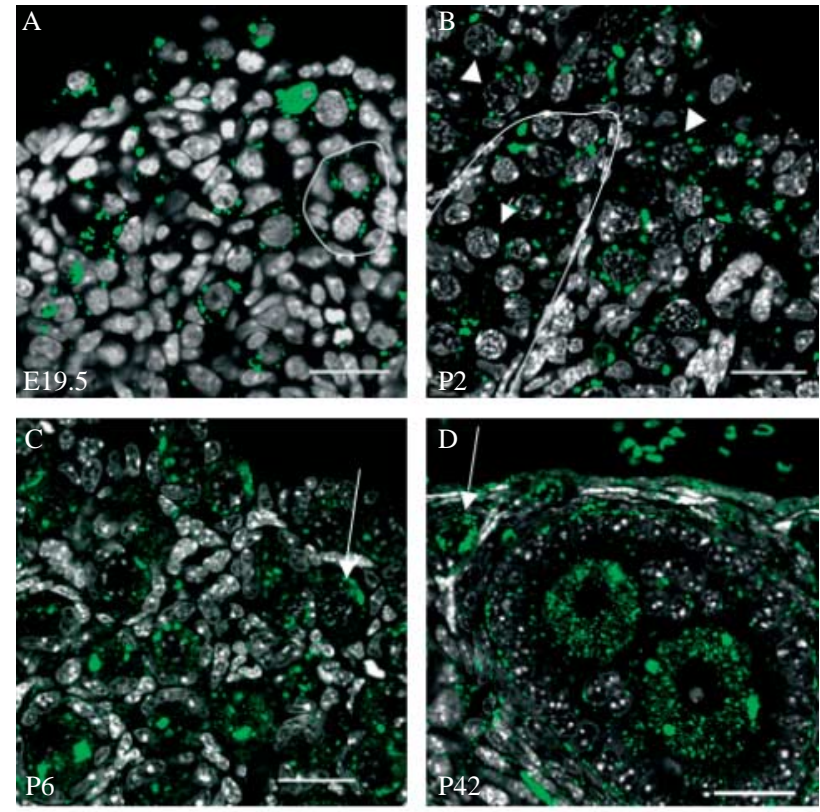

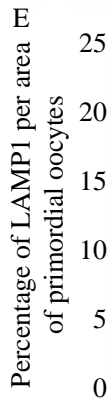
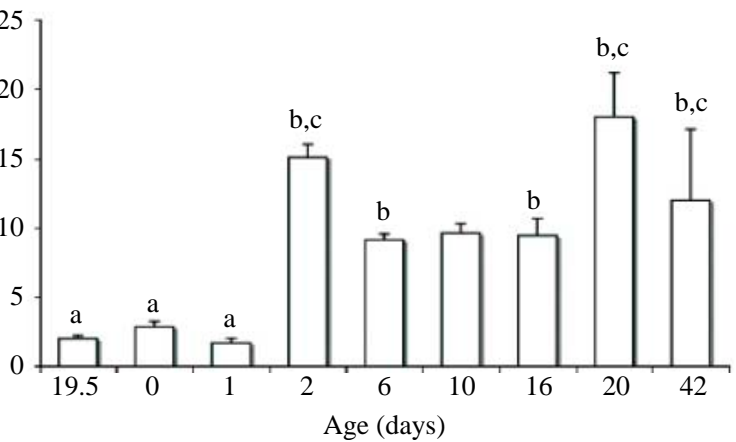

Figure 5 LAMP1 expression in neonatal mouse ovaries. Prenatal ovaries (A and B) exhibit LAMP1 stained lysosomes (green) predominantly in germ cells (somatic and germ cell nuclei are white). After birth, large lysosomes are evident in oocytes that form primordial follicles (C-D, arrows) whereas smaller lysosomes within oocytes that are not fully enclosed by follicle cells (B, arrowheads). (E) Shows quantitation of LAMP1 staining in oocytes between E19.5 and P42; note the abrupt increase at P2 and stabilization of lysosome density from P16 on. Columns with different letters are significantly different from each other $(P<0.05)$. Scale bars $=20 \mu \mathrm{m}$.

development (Fig. 6C and D). We next tested the effect of serum starvation on the loss of germ cells in an ovarian organ culture system.

To accomplish this, ovaries from P0 animals were cultured and exposed to LTR in the presence or absence of serum for $24 \mathrm{~h}$. Complete $\mathrm{Z}$ stack projections were thresholded to demarcate acidified germ cells (Fig. 7A-C and Supplementary Figure 2, which can be viewed online at www.reproduction-online.org/supplemental/), which were counted and expressed as mean density volume per ovary. Direct comparison between P0 (56.93 $\pm 8.86 ; \mathrm{Cl}: 18.80-95.08)$ and P1 (mean of 20.99) for ovaries showed a $60 \%$ decrease at these time points with freshly isolated tissues. Comparison of
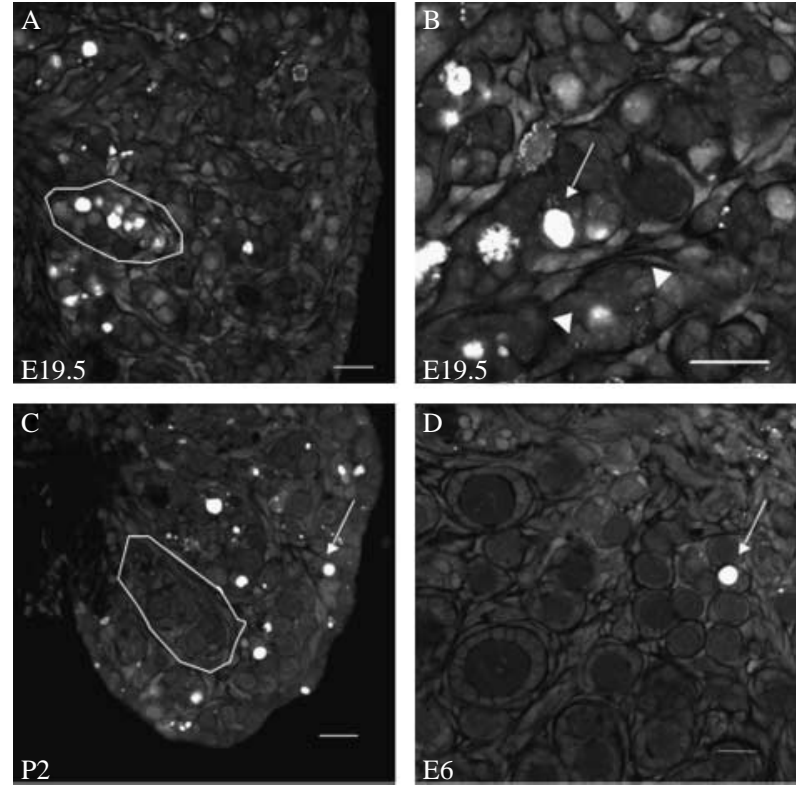

Figure 6 Analysis of LTR staining in intact mouse ovaries. E19.5 ovaries (A and B) just prior to birth containing both cytoplasmic acidification (arrows) and LTR foci (arrowheads) are seen within germ cell cluster (some circled for easy visualization). From P2 (C) to P6 (D), seem to have fewer cells with cytoplasmic acidification (arrows). Scale bars $=20 \mu \mathrm{m}$.

serum treated $(14.40 \pm 3.64 ; \mathrm{Cl}:-15.41-44.22)$ vs serum starved (23.61 $\pm 5.08 ; \mathrm{Cl}: 1.77-45.45)$ organ cultures illustrates a higher proportion of acidified germ cells after serum removal (Fig. 7G). These findings indicate that maintaining newborn ovaries in the presence of serum lessens the incidence of autophagy. We next tested the effects of apoptosis or autophagy inhibitors on the density of LTR positive germ cells.

\section{Autophagy as an effector of germ cell loss in the newborn ovary}

3-Methyl adenine (3MA), a commonly used autophagy inhibitor (Seglen \& Gordon 1982, Boya et al. 2005), and ZVAD, a commonly used pan-caspase inhibitor (Kim et al. 2001, Boya et al. 2005), were used to selectively impair autophagy or apoptosis in the organ culture system described above. Ovaries $(n=3)$ were exposed to either inhibitor alone or a combination of both inhibitors for $24 \mathrm{~h}$ and processed for LTR staining. In the presence of $3 \mathrm{MA}$, an apparent reduction in the density of acidified cells was evident. In contrast, ZVAD treatment had little discernible effect on the density of acidified cells compared to control ovaries (Fig. 7E and $\mathrm{C}$ respectively and Supplementary Figure 2). Interestingly, exposure to both $3 \mathrm{MA}$ and ZVAD (Fig. 7F) for $24 \mathrm{~h}$ reduced LTR-positive cell density to levels comparable with those observed in the 3MA treatment group (Fig. 7D and Supplementary Figure 2). These trends were confirmed by quantitative 

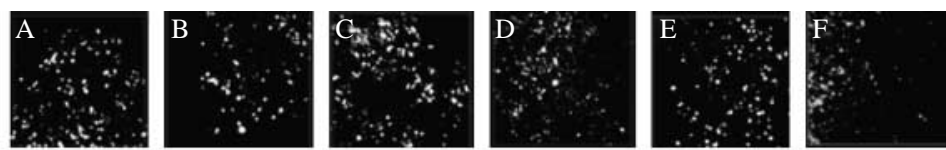

G

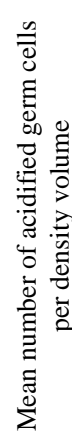

Complete media $\square$ DMEM only image analysis (Fig. 7G). Whereas the density of LTR-positive cells was similar in control $(24 \mathrm{~h}$, 23.61 $\pm 5.08 ; \mathrm{Cl}: 1.77-45.45)$ and ZVAD (28.77 \pm 8.86 ; $\mathrm{Cl}:-9.36-66.90)$ groups, $3 \mathrm{MA}$ alone $(12.05 \pm 3.45$; Cl: $-2.79-26.90)$ or in combination with ZVAD (13.38 $\pm 1.28 ; \mathrm{Cl}:-2.89-29.85)$ resulted in cell densities comparable with those attained after culture in the presence of serum (14.41 $\pm 3.64 ; \mathrm{Cl}$ : $-15.41-44.22)$. Collectively, these results indicate that inhibition of autophagy and not apoptosis influence germ cell loss in newborn ovaries. This was further supported by an analysis of protein expression in perinatal ovaries (Fig. 8). Mediators of apoptosis (caspase 3, cathepsin D) and autophagy (LC-3B and beclin 1) were probed by western blots of pooled ovary samples from animals of E19.5, P2, P6 and P20 ages. Figure 8 shows active

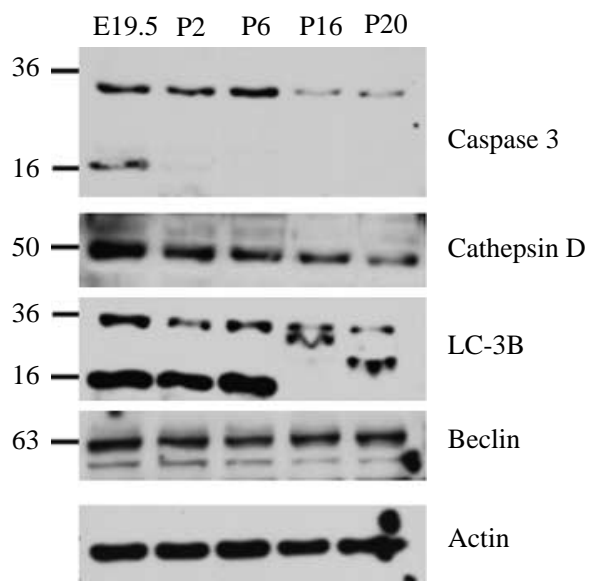

Figure 8 Apoptotic and autophagic protein expression. Western blot analysis of caspase 3 and cathepsin D (apoptosis), LC-3B and beclin 1 (autophagy), and $\beta$-actin expression in E19.5, P2, P6 and P20 pooled ovaries. caspase 3 protein expression only on E19.5 ( 17 kDa), and inactive caspase 3 expression $(32 \mathrm{kDa})$ through all ages examined. Expression of the $16 \mathrm{kDa} \mathrm{LC}-3 \mathrm{~B}$, the autophagosomal membrane form of LC3B (Tanida et al. 2005), was evident between E19.5 and P6. The $36 \mathrm{kDa}$ form of LC3B decreases at birth and remains relatively stable in expression at the later time points. Interestingly, cathepsin D gradually decreases until P20. Beclin levels show a slight decline at birth but also remain stable through to P20. Collectively, these data indicate that mediators of apoptosis and autophagy are differentially expressed while pre- and postnatal germ cell loss takes place.

\section{Discussion}

Most female eutherian mammals establish a stockpile of follicle-enclosed oocytes at or soon after birth that is gradually depleted through the organisms' reproductive lifespan. In such species, it has long been appreciated that the most profound period of germ cell loss occurs shortly after parturition (Faddy \& Gosden 2007). Moreover, it is generally held that the primary determinant of germ cell survival involves parallel processes of germ cell enclosure in the primordial follicle and selective susceptibility of some oocytes to type I PCD, apoptosis (Coucouvanis et al. 1993, De Pol et al. 1997, Reynaud \& Driancourt 2000, Pepling \& Spradling 2001, Tilly 2001). Since oocyte attrition is a fundamental tenet of reproductive aging and certain fertility disorders in women, there has been general interest in the mechanisms responsible for both the establishment and maintenance of the female germ cell supply (Gosden 2002, Matzuk 2005, Skinner 2005). Here, we used the commonly studied C57Bl/6 mouse strain to gain insight into the mechanisms of perinatal germ cell loss. While our results concur with previous studies indicating 
that substantial germ cell attrition occurs at the onset of reproductive maturity and birth, they also question type I PCD as the sole cause for early germ cell loss. Instead, multiple perinatal mechanisms (apoptosis, germ cell extrusion, autophagy) interact to establish a finite follicle reserve for later use. The conclusion that different PCD mechanisms operate at discrete stages of ovarian development suggests a level of complexity in the regulation of ovarian germ cell and follicle survival that has only recently been appreciated (Lobascio et al. 2007).

\section{Ovarian developmental transitions engage distinct PCD mechanisms}

Unlike earlier histomorphometric analysis of germ cells and follicles in the mouse ovary, our methods using immunostaining of $\mathrm{MVH}$ for unequivocal germ cell identification provided an accurate counting method for analysis of all follicle stages from E15.5 to P150 (Fig. 2). This kind of analysis confirmed the sharp decline in oocyte number at birth noted by others (Baker 1966, Coucouvanis et al. 1993, Ratts et al. 1995, McClellan et al. 2003, Kerr et al. 2006) and further showed that over the postnatal period approaching puberty (P6-P42), little change in follicle numbers was detected. Our results are in agreement with those of Kerr et al. (2006) (Supplementary Table 1). Many studies have demonstrated the occurrence of germ cell loss through apoptosis in fetal mouse ovary (Coucouvanis et al. 1993, Pepling \& Spradling 2001, De Felici et al. 2005) and in some cases have linked type I PCD to errors in meiotic cell cycle checkpoint control (Burgoyne \& Baker 1985). It should be emphasized, however, that the frequency of TUNEL-positive oocytes seen in fetal mouse ovary is surprisingly small ranging from 0.5 to $5 \%$ (Pesce et al. 1997, Pepling \& Spradling 2001). A recent re-examination of this stage of ovarian development by Lobascio et al. (2007) has concluded that previous studies failed to recognize the complexity of germ cell loss at fetal stages. Interestingly, these authors called attention to the fact that the mTOR inhibitor, rapamycin, increased TUNEL staining in germ cells presumably due to the negative effect of mTOR on autophagy. Moreover, calpain inhibitor 1 also induced apoptosis in germ cells after prolonged culture as evidenced by the appearance of caspase-negative and atypical TUNEL-positive oocytes (De Felici et al. 2007, Lobascio et al. 2007). The rare occurrence of apoptotic cells in perinatal ovaries described here further demonstrates that additional, PCD mechanisms, such as autophagy, are involved with germ cell attrition prior to and after birth in the mouse. These findings are immediately relevant to strategies seeking to prevent germ cell loss due to therapeutic or genetic causes and emphasize the need to clearly identify the PCD mechanisms involved.

\section{Germ cell extrusion during ovarian morphogenesis}

Oocytes have been noted to reside close to or within the ovarian epithelium in previous studies (Wordinger et al. 1990, Hirshfield 1992, Motta et al. 2003, Albertini \& Barrett 2004) but the relative rarity of these and their disappearance with further postnatal development of the ovary have led most to conclude that this is a minor cause of germ cell loss (Byskov 1982, Hirshfield 1992, Motta et al. 2003). Estimates based on the present studies would tend to support this idea based upon analysis of static images at various time points. While this process could result in loss of a significant fraction of oocytes resident at the time of birth, a definitive assessment awaits development of technologies suitable for an analysis of this dynamic process.

\section{Autophagy: an alternative mechanism for perinatal germ cell loss}

The perinatal period of life in placental mammals marks an abrupt transition in energy homeostasis. Mounting evidence shows that up regulation of autophagy follows in the immediate hours of postnatal life in many tissues and organs (Kuma et al. 2004). It is now accepted that this burst of autophagy is an adaptive response to nutritional stress once the newborn organism is deprived of placental nutrients (Kuma et al. 2004). Coupled with studies on fetal ovaries cited above (De Felici et al. 2007, Lobascio et al. 2007), our results implicate autophagy in the establishment of the follicle reserve. That multiple PCD mechanisms would be involved in this process is not surprising since it has been shown in many other systems (Kuma et al. 2004). Moreover, ultrastructural studies of mammalian oocytes have repeatedly documented the presence of lysosomes and autophagosomes in many species (Hertig \& Adams 1967, Wassarman \& Albertini 1994) but the developmental time course of their appearance and its relationship to autophagy have not been established.

Lysosome amplification is a hallmark of autophagy (Bursch 2001). Using LAMP1 immunocytochemistry and vital markers for lysosomes, we document amplification of lysosomes in oocytes upon birth (Fig. 5). Moreover, accumulation of lysosomes was most apparent in oocytes enclosed within primordial follicles whereas these structures were less apparent in oocytes that were either extruded through the ovarian surface or undergoing PCD.

However, discriminating between germ cells actively undergoing autophagy or apoptosis required application of a vital staining method that would permit assessment of germ cell behavior at critical developmental stages. For this, we used the LTR method and confocal microscopy so that intact living ovaries could be evaluated by digital image analysis (Fig. 6). Fetal and neonatal ovaries exhibited cytoplasmic acidification of germ cells that were characterized by their size and disposition. These 
germ cells were distinct from resident macrophages and smaller germ cells that exhibited fibrillar staining after LTR labeling. Quantitation of germ cells at (P0) and just after birth (P1) indicated a gradual decline in their density at this transition. Importantly, ovaries from newborn animals cultured in the presence of serum-enriched medium displayed fewer LTR positive germ cells than those maintained in a serum-free environment This finding suggests that the removal of ovary from a growth factorrich environment, rather than an endogenous signal, was able to elicit germ cell death at the time when major loss is occurring. Growth factors are known to prevent follicle loss in both in vivo and in vitro models of ovarian development (De Felici 2000, Nilsson \& Skinner 2001, Skinner 2005, Bristol-Gould et al. 2006) and one likely candidate for maintaining germ cells is Kit ligand (Parrott \& Skinner 1999, De Felici 2000, Hutt et al. 2006). While this experimental approach would be helpful in defining the physiological factors that regulate follicle formation and survival under dynamic conditions, some studies involving section analysis have supported the general notion that growth factor deprivation at birth is responsible for much of the germ loss seen at this time (Durlinger et al. 1999, Bristol-Gould et al. 2006). Whether the effects of serum on germ cell loss were mediated by apoptosis or autophagy was next investigated using selective inhibitors for each of these processes.

As shown above (Fig. 7 and Supplementary Figure 2), the selective autophagy inhibitor 3MA (Seglen \& Gordon 1982, Boya et al. 2005) reduced the acidified germ cell density compared to controls whereas no effect was seen after treatment with ZVAD, a pan-caspase inhibitor. Moreover, use of both inhibitors reduced the density of acidified germ cells to the same extent as 3MA alone, suggesting further that autophagy rather than apoptosis is involved in germ cell loss after birth. That combinatorial mechanisms may mediate this process is further indicated by western blot analysis of components for either apoptosis or autophagy (Fig. 8).

Prominent perinatal expression of beclin 1 and LC-3B (16 kDa) in ovaries at E19.5, P2 and P6 directly implicate autophagy, but, as also shown, activated caspase 3 $(\sim 17 \mathrm{kDa})$ is present only in fetal ovaries. Since cathepsin D is also present over this time interval, it is tempting to suggest that apoptosis at low levels may occur both prior to and following birth whereas the activation of autophagy appears to be directly linked to parturition-induced starvation. Caspase 3 antibody also detects a $36 \mathrm{kDa}$ component that most likely is the inactive, constitutively expressed form that requires cleavage for protease activation (Boone \& Tsang 1998). Moreover, $16 \mathrm{kDa}$ band corresponding to the LC3B II isoform is localized to autophagosomes and autolysosomes (Tanida et al. 2005). Together, these results support the existence of combinatorial mechanisms for germ cell loss and survival at birth. Further resolution of the interactions between both of these PCD pathways will be facilitated by the use of conditional knockouts of genes involved in each of these processes.

In summary, these results suggest that multiple PCD mechanisms function in the perinatal mouse ovary to establish the primordial follicle reserve. The relative contributions of apoptosis and autophagy may vary at distinct stages of ovarian development. The present findings will be of relevance to emergent technologies seeking to optimize and preserve high quality oocytes for experimental or clinical use. Furthermore, they uncover a level of complexity in female germ line development in eutherian mammals not previously appreciated. Future studies seeking to rescue female germ cells in newborns, adolescents or adults will need to take into account the diverse mechanisms that are at play during different stages in the lifespan of the mammalian ovary.

\section{Materials and Methods}

\section{Animals}

Inbred $\mathrm{C} 57 \mathrm{Bl} / 6$ mice (Charles River, or Taconic Farms Inc., Germantown, NY, USA) used for these experiments were housed in a $14 \mathrm{~h}$ light: $10 \mathrm{~h}$ dark environment at constant temperature. Food and water were provided ad libitum. All animals were killed by cervical dislocation. Mice were maintained and used in accordance with the policies of the University of Kansas Animal Care and Use Committee (protocol \# 2007-1681).

\section{Ovary collection and tissue preparation}

Right and left ovaries from each animal were collected at embryonic (E15.5 and E19.5), prepubertal (P2, 6, 10, 12, 16, 20 days of age) and adult (P42, 100 and 150 days of age) stages. Five ovaries were collected at each stage from at least two separate litters. Intact bursa enclosed ovaries were fixed in either Bouin's fluid (Sigma; follicle counts) or $2 \%$ paraformaldehyde (PFA, Sigma; immunohistochemistry), for 4-6 h at room temperature and overnight at $4{ }^{\circ} \mathrm{C}$ respectively. Following fixation, ovaries were transferred to $70 \%$ ethanol and processed for paraffin embedding by standard techniques. For whole mount analysis, intact ovaries were fixed overnight in $2 \%$ formaldehyde microtubule stabilization buffer (MTSB-XF), (Messinger \& Albertini 1991) at $4{ }^{\circ} \mathrm{C}$ and stored at $4{ }^{\circ} \mathrm{C}$ in a blocking buffer as previously described until use.

\section{Immunocytochemistry}

For follicle counts $5 \mu \mathrm{m}$ sections were dewaxed and re-hydrated using conventional methods. Endogenous peroxidases were quenched in $0.3 \%$ hydrogen peroxide in methanol (5 min, room temperature). After three washes in Automation Buffer (Biomeda-Fisher, Foster City, CA, USA), slides were blocked for $30 \mathrm{~min}$ at room temperature in 9\% goat serum (Zymed) containing 3\% BSA (Sigma). Sections were labeled (overnight, $4{ }^{\circ} \mathrm{C}$ ) with polyclonal rabbit 
antibody against $\mathrm{MVH}$, (a cytoplasmic germ cell specific protein, gift from Dr Noce) diluted 1:1200 in 1\% BSA. Washed sections were then incubated with biotinylated goat anti-rabbit serum (Zymed; 1:200 in 1\% BSA) for $30 \mathrm{~min}$ at room temperature, rinsed and incubated with HRP Avidin D (HRP; Vector, Burligame, CA, USA; 1:500) for $10 \mathrm{~min}$. Diaminobenzidine was used as a substrate for HRP. Sections were counterstained with PAS reagent and Harris hematoxylin (Protocol) containing $4 \%$ acetic acid. Tissues were dehydrated, cleared and coverslipped using Permount (Fisher, Pittsburgh, PA, USA). Control slides were processed identically but primary antibody was omitted.

\section{Follicle morphology and classification}

Follicles were classified according to Pedersen \& Peters (1968) and Myers et al. (2004). Briefly, follicles were classified as primordial if the oocytes were partially or completely surrounded by squamous GCs, equivalent to Type 1-2 in the Pedersen \& Peters (1968) classification. Primary follicles were those exhibiting one complete layer of cuboidal GCs, (Type $3-3 b)$. In the transition from primordial to primary classification was made according to the predominant type of GCs present. Secondary follicles were classified as all follicles having more than one GC layer and no visible antrum (Type 4-5). Follicles with a small antrum were designated early antral (Type 6) and antral follicles (Type 7) when the follicle had a single large antral space.

\section{Follicle counting}

Counts were made using a $40 \times$ Nikon objective in an Alphaphot 2 SY2 Nikon microscope. Follicles with an intact oocyte nucleus containing a dark nucleolus were counted in every second section and repeat scoring was unlikely because $\sim 12 \mu \mathrm{m}$ germ cells in primordial and primary follicles would not be present in the third $8 \mu \mathrm{m}$ section used for sampling frequency in this study. For these follicle classes, final totals were derived by doubling the follicles recorded in every second section. However, for secondary to pre-ovulatory follicles this adjustment was omitted in order to limit overrepresentation (Liu et al. 2002). The position of primordial follicles relative to the ovarian surface epithelium was also determined and these were scored as extra-ovarian (if in the bursal cavity) or intraepithelial (if associated with or subjacent to the epithelium).

\section{Static and dynamic imaging of germ cell loss}

PCD was studied using a combination of methods to discriminate between apoptosis and autophagy in histological samples or ovarian whole mounts.

\section{Apoptosis detection}

TUNEL was used to detect apoptosis following the manufacturer's protocol for the ApopTag Peroxidase In Situ apoptosis detection kit (Chemicon-Millipore, Billerica, MA, USA).
For active caspase 3 (1:100; R\&D Systems, cat. \# AF835), and poly (ADP ribose) polymerase (cleaved-PARP form; 1:50; AbCam cat. \# ab32064) paraffin sections of PFA fixed ovaries were dewaxed and microwaved (high, $15 \mathrm{~min}$ ) in sodium citrate buffer $(0.01 \mathrm{M}, \mathrm{pH}$ 6.0) for antigen retrieval. After sections were washed and blocked they were incubated in primary antibody (see above). For fluorescent imaging, slides were exposed to Alexa-fluor goat anti-rabbit secondary antibodies (488 or 568; 1:800; Molecular Probes, Invitrogen; $\left.37^{\circ} \mathrm{C} ; 1 \mathrm{~h}\right)$ rinsed $(3 \times)$, incubated in Hoechst $33258(1 \mu \mathrm{g} / \mathrm{ml}$; Polysciences Inc., Warrington, PA, USA) for nuclear staining and mounted in Prolong Anti-fade Reagent (Molecular Probes, Invitrogen). Control slides were prepared as above by omitting primary antibody.

Whole mount preparations of intact or fragments of ovaries fixed in MTSB-XF (Messinger \& Albertini 1991) were processed for confocal microscopy using the same primary and secondary antibodies noted above. These were diluted with wash solution and used with constant agitation for $24 \mathrm{~h}$ at $4{ }^{\circ} \mathrm{C}$. Multiple wash steps (a total of $4 \mathrm{~h}$, several changes; $37^{\circ} \mathrm{C}$ ) separated primary and secondary antibody incubations. Hoechst 33258 (nuclei) and Alexa 546 Phalloidin (f-actin; 1:100 dilution, Molecular Probes, Invitrogen) were used to define nuclear and cell boundaries. Ovaries were mounted on microscope slides in glycerol/PBS containing Hoechst 33258.

LTR is an aldehyde-fixable dye that concentrates in acidic membrane-bound intracellular compartments of living tissue, which has been adopted by Zucker et al. (1998) as an assay for PCD in rodent tissues. We modified the protocol as follows: intact ovaries were incubated in $5 \mu \mathrm{M}$ LTR (Molecular Probes, Invitrogen) in MEM (Gibco, Invitrogen) for 1 to $2 \mathrm{~h}$ at $37^{\circ} \mathrm{C}$ and $5 \% \mathrm{CO}_{2}$. After two washes in PBS the ovaries were fixed in a mixture of $4 \%$ PFA and $1 \%$ glutaraldehyde (Sigma) in PBS ( $2 \mathrm{~h}$; $37^{\circ} \mathrm{C}$ with shaking, followed by $4{ }^{\circ} \mathrm{C}$, overnight), rinsed twice in PBS and dehydrated in a series of methanol/PBS solutions (50, 70, 95 and $100 \%-2 \times ; 15$ min each). Tissues were then cleared in a 1:2 mixture of benzyl alcohol to benzyl benzoate (BABB, Sigma). From absolute methanol, tissues were exchanged through $50 \%$ and $70 \%$ BABB-methanol and finally pure BABB solution ( $2 \mathrm{~h}$ each; at room temperature with constant agitation). For confocal microscopy, a single ovary was centrally positioned in a metal washer sealed by Permount (Fisher) to the center of a glass bottomed tissue culture dish (Delta T, Fisher). The depression was filled with BABB and a coverslip applied with Permount (Fisher).

\section{Lysosome detection and autophagy}

Both immunohistochemistry and live cell imaging were used to visualize the lysosomes and autophagic markers. Lysosome abundance and distribution in developing germ cells was determined using a rat antibody to LAMP1 (a lysosomal membrane glycoprotein; Developmental Studies Hybridoma Bank, University of lowa, 1:100, cat. \#1D4B), and goat antirat Alexa Fluor 488 secondary antibody in both paraffin sections and whole mounts as described above. For analysis of acidic organelles in living tissues, intact ovaries (embryonic 19.5 and post-natal days 0 (day of birth), 1 and 2) were cultured for 4 and $18 \mathrm{~h}$ in Acridine Orange $(1 \mu \mathrm{g} / \mathrm{ml}$; 
Molecular Probes, Invitrogen) or LysoSensor Yellow/Blue DND $(10 \mu \mathrm{g} / \mathrm{ml}$; Molecular Probes, Invitrogen). Individual ovaries were cultured in $100 \mu \mathrm{l}$ drops of culture media (MEM with Earle's Salts and L-glutamine without phenol red (Gibco); $10 \%$ FCS (Hyclone, Logan, UT, USA), $100 \mathrm{ng} / \mathrm{ml}$ of leukemia inhibitory factor (Sigma), $100 \mathrm{ng} / \mathrm{ml}$ of stem cell factor (SCF/c-Kit, PreProtech), $50 \mathrm{ng} / \mathrm{ml}$ of IGF1 (Sigma), and 1\% gentomycin (Sigma)), covered with mineral oil (Sigma) and incubated with at $37{ }^{\circ} \mathrm{C}$ with $5 \% \mathrm{CO}_{2}$. Glass bottom culture dishes allow live imaging of intact ovaries after 4 or $18 \mathrm{~h}$ of culture and Hoechst 33342 was used as a vital nuclear stain. LAMP1 was quantified using Integrated Morphometry Analysis (IMA) software (MetaMorph version 7.5 Universal Image Corporation, USA), to define individual germ cell boundaries (regions of interest) and measuring the area occupied by the LAMP1 signal. Values are represented as the percentage of LAMP1 per germ cell.

\section{In vitro starvation of neonatal ovaries}

To create in vitro conditions resembling the nutrient-deprived state of neonatal ovaries, P0 and P1 ovaries (3 per group from different litters) were incubated in $600 \mu \mathrm{l}$ complete organ culture media (DMEM:Ham's F12, 1:1; 10\% FBS, 1\% ITS (Sigma), 1\% penicillin/streptomycin (P/S, Sigma)), suspended in a $3 \mu \mathrm{m}$ PCF insert Millicell (Millipore), and placed in a 24 well tissue culture plate. Following a stabilization period, ovaries were either incubated for an additional $21 \mathrm{~h}$ in complete media or starvation media $(\mathrm{DMEM}+1 \% \mathrm{P} / \mathrm{S})$. At the end of the total $24 \mathrm{~h}$ incubation period, LTR $(1 \%)$ was added to each well. For $0 \mathrm{~h}$ control ovaries, LTR was added for $3 \mathrm{~h}$ following the initial stabilization period. Hoechst 33342 was typically added as a nuclear counterstain. Ovaries were washed in PBS and fixed as for LTR in 4\% PFA prior to confocal microscopy without BABB clearing.

\section{Effect of apoptosis and autophagy inhibitors on starved neonatal ovaries}

Using complete or starvation media (as above), E19.5 or P0 ovaries were incubated with inhibitors of PCD. For apoptosis the pan-caspase Inhibitor 1 ZVAD (OME)-FMK (50 $\mu \mathrm{M}$; Calbiochem-EMD, Gibbstown, NJ, USA) was used; for autophagy, 3MA (10 mM; Sigma) was used. Drugs were added alone or in combination for $21 \mathrm{~h}$ and LTR and Hoechst 33342 were added for the final $3 \mathrm{~h}$ of incubation. Tissues were analyzed by confocal microscopy to determine the volume density of LTR labeled germ cells in Z-section composites of whole ovaries using IMA software as above. Briefly, LTR positive objects with a diameter greater than $80 \mu \mathrm{m}^{2}$ (determined to be the approximate diameter of germ cells) were thresholded and counted within 3-dimensional projections of a single ovary from each treatment group ( $n=3$ per group). Approximately, $50 \%$ of the entire ovary volume was assayed for each replicate in each treatment group and the data are expressed as mean density volume per group.

\section{Western blot analysis of apoptosis and autophagy proteins}

Ovaries from fetal (E19.5), neonatal (P0, P1, P2, and P6), and pubertal (P20) mice were washed in cold PBS $\left(4{ }^{\circ} \mathrm{C}\right)$, and lysed (20 mM Tris- $\mathrm{HCl}$ (pH 7.4; Sigma), $150 \mathrm{mM}$ of sodium chloride (Sigma), $1 \mathrm{mM}$ of EDTA (Sigma), $1 \mathrm{mM}$ EGTA (Sigma), 1\% Triton, $2.5 \mathrm{mM}$ sodium pyrophosphate (Sigma), $1 \mathrm{mM} \beta$-glycerolphosphate, $1 \mathrm{mM}$ sodium orthovanadate (Sigma), $1 \mu \mathrm{g} / \mathrm{ml}$ leupetin (Sigma), $1 \mathrm{mM}$ phenylmethanesulfonyl fluoride (Sigma)). Twenty micrograms of protein from pooled samples (E19.5, P2, 6, 16, and 20) was loaded on $12 \%$ SDS-Page gels and transferred to a PVDF membrane (Millipore). Membranes were incubated for $1 \mathrm{~h}$ in PBSTween $20(0.05 \%)$ containing 5\% nonfat milk and probed with primary antibodies to caspase 3 (Cell Signaling cat. \# 9661), LC-3B (Cell Signaling cat. \# 2775), cathepsin D (Santa Cruz, cat. \# 10725) and beclin 1 (Sigma cat. \# B6061) followed by incubation with the appropriate HRP-conjugated secondary antibody (Southern Biotechnologies Associates, Birmingham, AL, USA); bands were detected with SuperSignal West Pico chemiluminescent substrate (Pierce, Rockford, IL, USA) and an anti-actin antibody (Sigma cat. \# A2066) was used to verify comparable loading between samples.

\section{Image acquisition and analysis}

Whole mount or sectioned ovary preparations were imaged using a LSM-510 Pascal confocal microscope (Zeiss) mounted on a Zeiss Axiovert 200M microscope equipped with excitation specific Diode (405 nm), Argon (458, 477, 488 and $514 \mathrm{~nm}$ ) and Helium Neon lasers (594 nm). Single scans or Z series data sets were made using $20 \times, 40 \times$ and $63 \times(N A=1.25)$ objectives and data were archived and analyzed using LSM software. Bright field images were obtained using a Nikon Eclipse $8 \mathrm{i}$ microscope with $20 \times$ and $100 \times$ objectives and a Sony XWaveHAD color camera.

\section{Statistical analysis}

Data are presented as mean \pm S.E.M. and statistical analysis of follicle number counts was performed using Prism 4.0 (GraphPad Software Inc., San Diego, CA, USA). Data were analyzed by calculation of $\mathrm{Cl}$ and non-parametric KruskalWallis test of significance. Data were considered statistically significant in cases where $P<0.05$.

\section{Declaration of interest}

The authors declare that there is no conflict of interest that could be perceived as prejudicing the impartiality of the research reported.

\section{Funding}

This work was funded by: The Hall Family Foundation, The ESHE Fund, Fundação para a Ciência e Tecnologia (SFRH/BD/6439/2001), Fundação Calouste Gulbenkian (nr 78591), and NIH grant HD042076. 


\section{Acknowledgements}

We would like to thank Lane Christenson, Xiaoman Wong and John Bromfield for their assistance with the western blot experiments, Dr T Noce (Japan) for his generous gift of $\mathrm{MVH}$ antibody, and the Albertini Laboratory for their continued support and suggestions.

\section{References}

Albertini DF \& Barrett SL 2004 The developmental origins of mammalian oocyte polarity. Seminars in Cell and Developmental Biology 15 599-606.

Baker TG 1966 A quantitative and cytological study of oogenesis in the rhesus monkey. Journal of Anatomy 100 761-776.

Boone DL \& Tsang BK 1998 Caspase-3 in the rat ovary: localization and possible role in follicular atresia and luteal regression. Biology of Reproduction 58 1533-1539.

Boya P, Gonzalez-Polo RA, Casares N, Perfettini JL, Dessen P, Larochette N, Metivier D, Meley D, Souquere S, Yoshimori T et al. 2005 Inhibition of macroautophagy triggers apoptosis. Molecular and Cellular Biology 25 1025-1040.

Bristol-Gould SK, Kreeger PK, Selkirk CG, Kilen SM, Cook RW, Kipp JL, Shea LD, Mayo KE \& Woodruff TK 2006 Postnatal regulation of germ cells by activin: the establishment of the initial follicle pool. Developmental Biology 198 132-148.

Burgoyne PS \& Baker TG 1985 Perinatal oocyte loss in XO mice and its implications for the aetiology of gonadal dysgenesis in $\mathrm{XO}$ women. Journal of Reproduction and Fertility 75 633-645.

Bursch W 2001 The autophagosomal-lysosomal compartment in programmed cell death. Cell Death and Differentiation 8 569-581.

Byskov AG 1982 Primordial Germ Cells and Regulation of Meiosis, Cambridge, MA: Cambridge University Press.

Coucouvanis EC, Sherwood SW, Carswell-Crumpton C, Spack EG \& Jones PP 1993 Evidence that the mechanism of prenatal germ cell death in the mouse is apoptosis. Experimental Cell Research 209 238-247.

Durlinger AL, Kramer P, Karels B, de Jong FH, Uilenbroek JT, Grootegoed JA \& Themmen AP 1999 Control of primordial follicle recruitment by anti-Mullerian hormone in the mouse ovary. Endocrinology 140 5789-5796.

Edinger AL \& Thompson CB 2004 Death by design: apoptosis, necrosis and autophagy. Current Opinion in Cell Biology 16 663-669.

Faddy M \& Gosden R 2007 Numbers of ovarian follicles and testing germ line renewal in the postnatal ovary: facts and fallacies. Cell Cycle $\mathbf{6}$ 1951-1952.

De Felici M 2000 Regulation of primordial germ cell development in the mouse. International Journal of Developmental Biology 44 575-580.

De Felici M, Klinger FG, Farini D, Scaldaferri ML, Iona S \& Lobascio M 2005 Establishment of oocyte population in the fetal ovary: primordial germ cell proliferation and oocyte programmed cell death. Reproductive Biomedicine Online 10 182-191.

De Felici M, Lobascio AM \& Klinger FG 2007 Cell death in fetal oocytes: many players for multiple pathways. Autophagy 4 240-242.

Fujiwara $Y$, Komiya $T$, Kawabata $H$, Sato $M$, Fujimoto $H$, Furusawa $M$ \& Noce T 1994 Isolation of a DEAD-family protein gene that encodes a murine homolog of Drosophila vasa and its specific expression in germ cell lineage. PNAS 91 12258-12262.

Gosden RG 2002 Oogenesis as a foundation for embryogenesis. Molecular and Cellular Endocrinology 186 149-153.

Guicciardi ME, Leist M \& Gores G) 2004 Lysosomes in cell death. Oncogene 23 2881-2890.

Hertig AT \& Adams EC 1967 Studies on the human oocyte and its follicle. I. Ultrastructural and histochemical observations on the primordial follicle stage. Journal of Cell Biology 34 647-675.

Hirshfield AN 1992 Heterogeneity of cell populations that contribute to the formation of primordial follicles in rats. Biology of Reproduction $\mathbf{4 7}$ 466-472.
Hutt KJ, McLaughlin EA \& Holland MK 2006 KIT/KIT ligand in mammalian oogenesis and folliculogenesis: roles in rabbit and murine ovarian follicle activation and oocyte growth. Biology of Reproduction $\mathbf{7 5}$ 421-433.

Kerr JB, Duckett R, Myers M, Britt KL, Mladenovska T \& Findlay JK 2006 Quantification of healthy follicles in the neonatal and adult mouse ovary: evidence for maintenance of primordial follicle supply. Reproduction 132 95-109.

Kim SO, Ono K \& Han J 2001 Apoptosis by pan-caspase inhibitors in lipopolysaccharide-activated macrophages. American Journal of Physiology. Lung Cellular and Molecular Physiology 281 L1095-L1105.

Kuma A, Hatano M, Matsui M, Yamamoto A, Nakaya H, Yoshimori T, Ohsumi Y, Tokuhisa T \& Mizushima N 2004 The role of autophagy during the early neonatal starvation period. Nature 432 1032-1036.

Liu J, Van der Elst J, Van den Broecke R \& Dhont M 2002 Early massive follicle loss and apoptosis in heterotopically grafted newborn mouse ovaries. Human Reproduction 17 605-611.

Lobascio AM, Klinger FG, Scaldaferri ML, Farini D \& De Felici M 2007 Analysis of programmed cell death in mouse fetal oocytes. Reproduction 134 241-252.

Lum JJ, DeBerardinis RJ \& Thompson CB 2005 Autophagy in metazoans: cell survival in the land of plenty. Nature Reviews. Molecular Cell Biology 6 439-448.

Maiuri MC, Zalckvar E, Kimchi A \& Kroemer G 2007 Self-eating and selfkilling: crosstalk between autophagy and apoptosis. Nature Reviews. Molecular Cell Biology 8 741-752.

Matzuk MM 2005 Gynecologic diseases get their genes. Nature Medicine $1124-26$.

McClellan KA, Gosden R \& Taketo T 2003 Continuous loss of oocytes throughout meiotic prophase in the normal mouse ovary. Developmental Biology 258 334-348.

Messinger SM \& Albertini DF 1991 Centrosome and microtubule dynamics during meiotic progression in the mouse oocyte. Journal of Cell Science 100 289-298.

Motta PM, Nottola SA, Familiari G, Makabe S, Stallone T \& Macchiarelli G 2003 Morphodynamics of the follicular-luteal complex during early ovarian development and reproductive life. International Review of Cytology 223 177-288.

Myers M, Britt KL, Wreford NG, Ebling FJ \& Kerr JB 2004 Methods for quantifying follicular numbers within the mouse ovary. Reproduction 127 569-580.

Nilsson E \& Skinner MK 2001 Cellular interactions that control primordial follicle development and folliculogenesis. Journal of the Society for Gynecologic Investigation 8 S17-S2O.

Parrott JA \& Skinner MK 1999 Kit-ligand/stem cell factor induces primordial follicle development and initiates folliculogenesis. Endocrinology 140 $4262-4271$.

Pedersen T \& Peters H 1968 Proposal for a classification of oocytes and follicles in the mouse ovary. Journal of Reproduction and Fertility 17 555-557.

Pepling ME \& Spradling AC 2001 Mouse ovarian germ cell cysts undergo programmed breakdown to form primordial follicles. Developmental Biology 234 339-351.

Perez GI, Robles R, Knudson CM, Flaws JA, Korsmeyer SJ \& Tilly JL 1999 Prolongation of ovarian lifespan into advanced chronological age by Bax-deficiency. Nature Genetics 21 200-203.

Pesce M, Di Carlo A \& De Felici M 1997 The c-kit receptor is involved in the adhesion of mouse primordial germ cells to somatic cells in culture. Mechanisms of Development 68 37-44.

De Pol A, Vaccina F, Forabosco A, Cavazzuti E \& Marzona L 1997 Apoptosis of germ cells during human prenatal oogenesis. Human Reproduction 12 2235-2241.

Qu X, Zou Z, Sun Q, Luby-Phelps K, Cheng P, Hogan RN, Gilpin C \& Levine B 2007 Autophagy gene-dependent clearance of apoptotic cells during embryonic development. Cell 128 931-946.

Ratts VS, Flaws JA, Kolp R, Sorenson CM \& Tilly JL 1995 Ablation of bcl-2 gene expression decreases the numbers of oocytes and primordial follicles established in the post-natal female mouse gonad. Endocrinology 136 3665-3668.

Reynaud K \& Driancourt MA 2000 Oocyte attrition. Molecular and Cellular Endocrinology 163 101-108. 
Seglen PO \& Gordon PB 1982 3-Methyladenine: specific inhibitor of autophagic/lysosomal protein degradation in isolated rat hepatocytes. PNAS 79 1889-1892.

Skinner MK 2005 Regulation of primordial follicle assembly and development. Human Reproduction Update 11 461-471.

Tanida I, Minematsu-Ikeguchi N, Ueno T \& Kominami E 2005 Lysosomal turnover, but not a cellular level, of endogenous LC3 is a marker for autophagy. Autophagy 1 84-91.

Tilly JL 2001 Commuting the death sentence: how oocytes strive to survive. Nature Reviews. Molecular Cell Biology 2 838-848.

Wassarman PM \& Albertini DF 1994 The Mammalian Ovum, New York, NY: Raven Press.

Wordinger R, Sutton J \& Brun-Zinkernagel AM 1990 Ultrastructure of oocyte migration through the mouse ovarian surface epithelium during neonatal development. Anatomical Record 227 187-198.
Yu L, Lenardo MJ \& Baehrecke EH 2004 Autophagy and caspases: a new cell death program. Cell Cycle 3 1124-1126.

Zucker RM, Hunter S \& Rogers JM 1998 Confocal laser scanning microscopy of apoptosis in organogenesis-stage mouse embryos. Cytometry 33 348-354.

Received 13 May 2008

First decision 23 June 2008

Revised manuscript received 16 January 2009

Accepted 27 January 2009 